\title{
Multi-Color Laser Spectroscopy with a Dual-Wavelength Quantum Cascade Laser
}

\author{
J. Jágerská ${ }^{* 1}$, P. Jouy ${ }^{2}$, B. Tuzson ${ }^{1}$, H. Looser ${ }^{3}$, A. Hugi ${ }^{2}$, M. Mangold ${ }^{1}$, P. Soltic ${ }^{1}$, J. Faist ${ }^{2}$, L. Emmenegger ${ }^{1}$ \\ ${ }^{I}$ Empa, Überlandstr. 129, 8600 Dübendorf, Switzerland \\ ${ }^{2}$ ETH-Zürich, Wolfgang-Pauli-Str.16, 8093 Zürich, Switzerland \\ ${ }^{3} \mathrm{FHNW}$, Klosterzelgstrasse 2, 5210 Windisch, Switzerland \\ jana.jagerska@empa.ch
}

\begin{abstract}
A new concept of multi-color spectroscopy based on a dual-wavelength QCL is presented. The latter emits at two distinct wavelengths $(5.26$ and $6.25 \mu \mathrm{m})$, featuring simultaneous detection of two different gas species without any beam combining optics.

OCIS codes: (300.6190) Spectrometers; (300.6360) Spectroscopy, laser; (140.3070) Infrared and far-infrared lasers; (140.5965) Semiconductor lasers, quantum cascade; (280.1740) Combustion diagnostics; (280.1120) Air pollution monitoring.
\end{abstract}

\section{Introduction}

Simultaneous detection of multiple gas species using MIR laser spectroscopy is highly desired for numerous applications ranging from air quality monitoring, medical breath analysis, drug and explosive detection to industrial process control. Since it is often impossible to address the spectra of different gases with a single laser, state-of-theart multi-color spectrometers have to rely on the use of several lasers and elaborate beam combining solutions. This makes them bulky, costly, and highly sensitive to optical alignment.

In this paper, we explore a completely new concept for multi-color spectroscopy based on a Dual-Wavelength Quantum Cascade Laser (DW-QCL) [1]. Such a laser can emit at two spectrally well-separated wavelengths, which share a common waveguide to produce one output beam. Thereby, it is possible to detect multiple gases with a single optical path, without the need for any beam combining optics.

\section{Dual-Wavelength Quantum Cascade Laser}

As schematically shown in Figure 1a), the active region of the DW-QCL consists of two different active layers stacked on top of each other, optimized for a broadband emission at $1600 \mathrm{~cm}^{-1}$ and $1900 \mathrm{~cm}^{-1}$. These two spectral windows are ideally suited for the detection of nitrogen oxide $(\mathrm{NO})$ and nitrogen dioxide $\left(\mathrm{NO}_{2}\right)$. Single-mode emission at the desired wavelengths is ensured by a succession of two distributed-feedback (DFB) gratings with different periodicities. Electrical separation of the respective laser sections makes it possible to address each wavelength independently.

a)
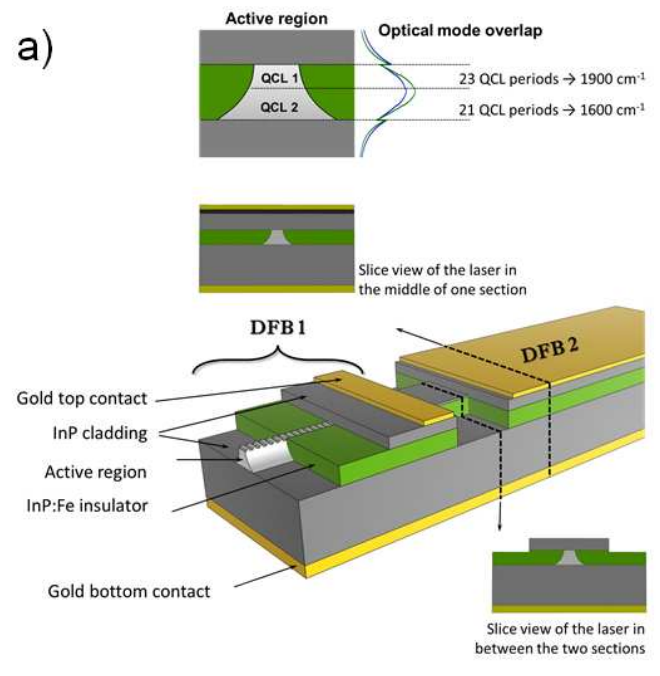

b)
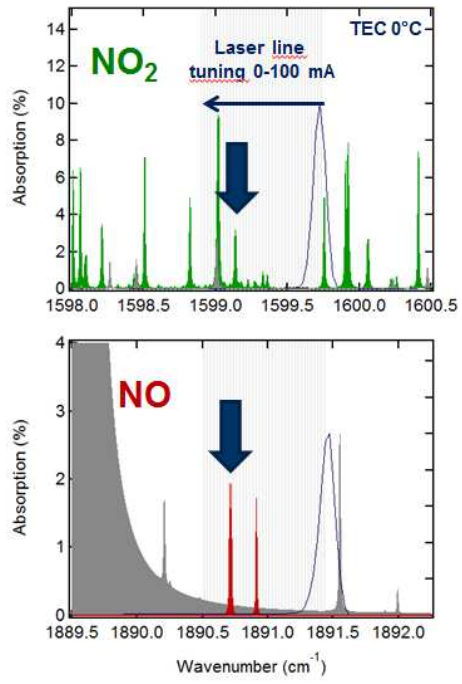

Fig. 1. a) Schematic drawing of a Twin QCL. b) Spectral emission properties of the Twin QCL at $0^{\circ} \mathrm{C}$ (grey shaded region) compared to the simulated absorption spectra of $\mathrm{NO}_{2}$ and $\mathrm{NO}$. The arrows mark the spectral lines selected for the $\mathrm{NO}_{2}$ and $\mathrm{NO}$ concentration measurement. 


\section{Multi-color spectroscopic system}

Integrated in a direct absorption laser spectroscopic setup, the spectral emission of the dual-wavelength QCL is time-division multiplexed at $100 \mathrm{~Hz}$ to account for quasi-simultaneous detection of both gases. Apart from a custom made multiplexer, the spectrometer is analogous to a single-color system with one laser driver, a 36-m multipass cell and a single MCT detector. The laser is operated in pulsed mode (the development of a continuous wave laser is ongoing) and tuned over several wavenumbers by applying a 0-100 mA sub-threshold current ramp. As shown in Figure 1b), the targeted species exhibit a good spectral overlap with the DW-QCL emission and, hence, spectral signatures of $\mathrm{NO}$ and $\mathrm{NO}_{2}$ are accessed simultaneously.

Custom written software controls and synchronizes the laser with a high-speed digitizer that acquires the data at $200 \mathrm{MS} / \mathrm{s}$ rate. A separate module of the program with a direct link to the HITRAN database [2] provides for realtime fitting of the absorption spectra, allowing for highly-sensitive $\mathrm{NO}$ and $\mathrm{NO}_{2}$ detection down to ppb levels.

\section{Application to $\mathrm{NO}$ and $\mathrm{NO}_{2}$ spectroscopy}

The prototype spectrometer was successfully tested both as a sensitive ambient air monitor, as well as for fast, $10 \mathrm{~Hz}$ operation in hash environment during automotive exhaust emission measurement. In terms of sensitivity, the system performance with a precision $1 \sigma=0.5 \mathrm{ppb}$ for $\mathrm{NO}_{2}$ and $1.5 \mathrm{ppb}$ for $\mathrm{NO}$ after $100 \mathrm{~s}$ of averaging was found comparable to other single-color pulsed QC laser systems [3]. The automotive emission test (see Figure 2) further confirmed that the spectrometer shows a very good accuracy within a wide range of concentration levels.

The presented result strongly indicates that the multi-wavelength QCL technology will soon set a new trend in multi-color spectrometer development.

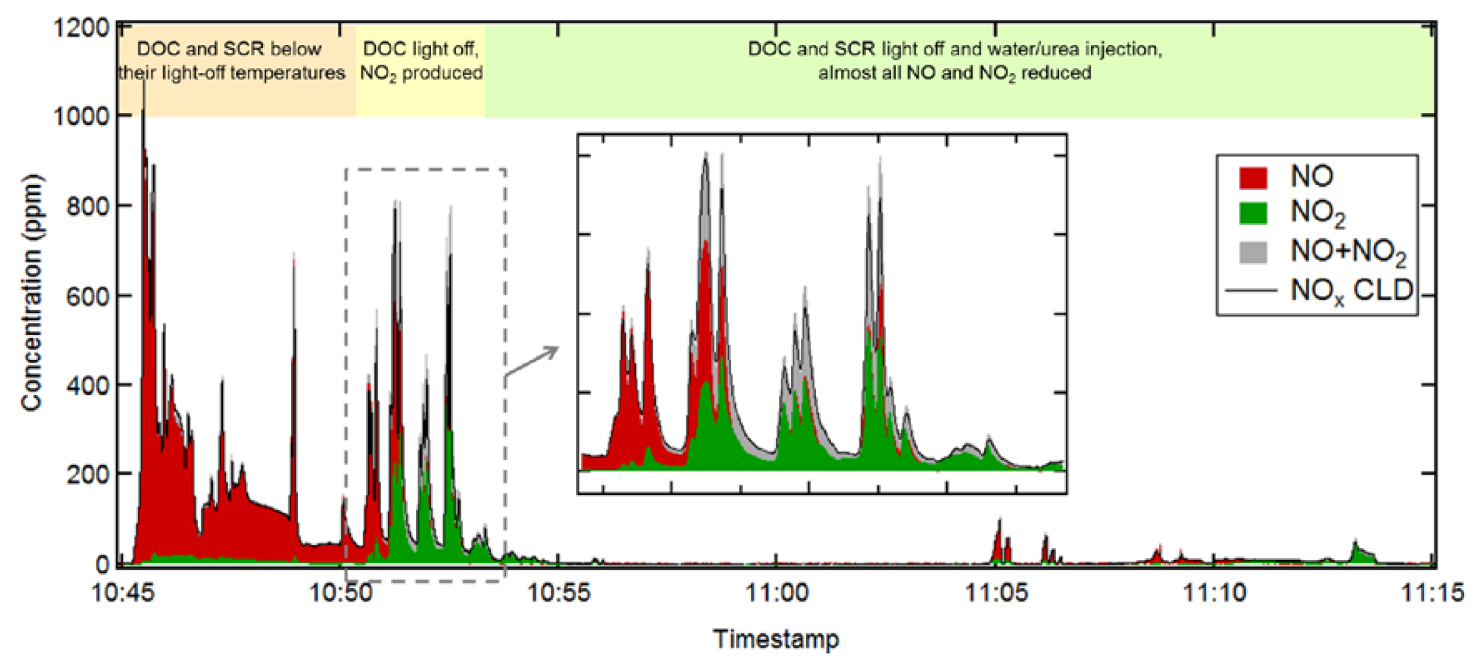

Fig. 2. $\mathrm{NO}$ (red) and $\mathrm{NO}_{2}$ (green) emissions measured by the DW-QCL spectrometer after the exhaust gas treatment system of a diesel engine, which was started at cooled down conditions and operated in a dynamic test cycle. Comparison of the sum of $\mathrm{NO}$ and $\mathrm{NO}_{2}$ with a standardized automotive chemiluminescence $\mathrm{NO}_{\mathrm{x}}$ detector is shown in grey.

[1] C. Gmachl, A. Straub, R. Colombelli, F. Capasso, D.L. Sivco, A.M. Sergent et al., "Single-mode, tunable distributed-feedback and multiplewavelength quantum cascade lasers," IEEE J. Quantum Elect. 38, 569-581 (2002).

[2] L.S. Rothman, I.E. Gordon, A. Barbe et al., "The HITRAN 2008 molecular spectroscopic database,” J. Quant. Spectrosc. Radiat. Transfer 110, 533-572 (2009).

[3] J. B. McManus, M. S. Zahniser, D. D. Nelson, Jr.,J. H. Shorter, S. Herndon, E. Wood and R. Wehr, “Application of quantum cascade lasers to high-precision atmospheric trace gas measurements," Opt. Eng. 49, 111124 (2010). 